SWEETHEART-Studie

\title{
Diabetikerinnen mit Herzinfarkt haben schlechte Prognose
}

\author{
Etwa die Hälfte aller Herzinfarkt-Patienten weisen einen \\ Diabetes mellitus auf. Frauen haben in solchen Fällen eine \\ ungünstigere Prognose als Männer.
}

- Herzkrankheiten und Diabetes treten häufig gemeinsam auf. Aus diesem Grund sollte bei Herzpatienten der Glukosestoffwechsel und bei Diabetes-Patienten das Herz untersucht werden. Die Koinzidenz Herzinfarkt/Diabetes untersuchte die deutsche SWEETHEART-Studie an 2767 Patienten. War kein Diabetes bekannt, wurde am vierten Tag nach Infarkt ein oraler Diabetes-Toleranztest durchgeführt.

Die Ergebnisse zeigten Geschlechtsunterschiede. Wie Prof. Diethelm Tschöpe, Herz- und Diabetes-Zentrum in Bad Oeynhausen berichtete, waren weibliche Infarkt-Patienten älter als männliche. Bei ihnen war häufiger ein Diabetes bekannt (30\% vs. $23 \%$ ), und die 3-Jahres-Mortalität war mit $30 \%$ etwa so hoch wie die der Männer (35\%). Bei neu entdeckten Diabetikern war aber die 3-Jahres-Mortalität bei Frauen deutlich höher als bei Männern ( $31 \%$ vs. $22 \%)$.

$\mathrm{DE}$.

Tod-im-Bett-Syndrom

\section{Woran sterben die junge Diabetiker?}

\section{Dem Tod-im-Bett-Syndrom junger Diabetiker liegt ver- mutlich eine Rhythmusstörung zugrunde, ausgelöst durch Unterzuckerung und autonome Neuropathie.}

- Typ-1-Diabetiker haben ein ca. dreifach erhöhtes Risiko für einen Sekundentod. Viele der jungen Diabetiker sterben nachts im Bett, daher der Name „Tod-im-Bett-Syndrom“ (Inzidenz 20-60 pro 100000 Patienten-Jahre).

Die Autopsie bestätigt oft eine Hypoglykämie. Diese kann zur QT-Verbreiterung und somit zu ventrikulären Arrhythmien führen, berichtete Prof. Simon Heller, Diabetologe an der Universität Sheffield. Demnach wäre die Kombination einer heftigen nächtlichen Hypoglykämie sowie eines geschädigten kardialen Reizleitungssystems aufgrund einer autonomen Neuropathie für die Rhythmusstörung verantwortlich. Es gelte, Risikopatienten zu erkennen, um sie mit Defibrillatoren zu schützen.

$\mathrm{DE}$.

- Quelle: 48th Annual Meeting of the European Association

for the Study of Diabetes, Berlin, Oktober 2012

\section{ACHTUNG:}

\section{Hier muss der Dummy durch eine Anzeige ersetzt werden !!}

